CAHIERS

MONDES

ANCIENS

\section{Cahiers « Mondes anciens »}

Histoire et anthropologie des mondes anciens

$13 \mid 2020$

Qu'est-ce que faire école? Regards sur « l'école de

Paris »

\title{
Notes de voyage en pays parisien
}

Travel Notes to the Parisian Land

\section{Gabriella Pironti}

\section{(2) OpenEdition}

Journals

Édition électronique

URL : http://journals.openedition.org/mondesanciens/2686

ISSN : 2107-0199

Éditeur

UMR 8210 Anthropologie et Histoire des Mondes Antiques

Référence électronique

Gabriella Pironti, « Notes de voyage en pays parisien », Cahiers « Mondes anciens » [En ligne], 13| 2020,

mis en ligne le 10 juin 2020, consulté le 12 juin 2020. URL : http://journals.openedition.org/ mondesanciens/2686

Ce document a été généré automatiquement le 12 juin 2020.

\section{(c) (i) (9)}

Les Cahiers «Mondes Anciens » sont mis à disposition selon les termes de la licence Creative Commons Attribution - Pas d'Utilisation Commerciale - Pas de Modification 4.0 International. 


\title{
Notes de voyage en pays parisien
}

\author{
Travel Notes to the Parisian Land
}

\section{Gabriella Pironti}

Une «École de Paris » n'a sans doute jamais existé sous la forme qu'on lui attribue, aujourd'hui encore, dans une partie du monde académique. Cette étiquette est employée, le plus souvent en dehors des milieux francophones, pour définir le groupe de chercheurs qui ont évolué autour de Jean-Pierre Vernant dans la seconde moitié du siècle dernier et ont contribué avec lui à renouveler en profondeur les études sur l'Antiquité classique, en les ouvrant notamment à l'anthropologie historique. Comme la plupart des étiquettes, elle est le fruit d'un regard extérieur et critique, et sa portée, dans l'acception des utilisateurs, n'est que très rarement dissociable d'une intention réductrice, autrement dit d'une volonté de constituer en objet unifié et identifiable un ensemble complexe d'idées et d'approches. Ce type d'opération consiste précisément à se forger ce que les Italiens appelleraient un «idolo polemico ». Un exemple révélateur à ce propos est offert par un passage d'un ouvrage assez récent sur la religion grecque antique $^{1}$ : le lexique et les images employés par l'auteur suggèrent qu'il assimile la Paris school à une sorte d'Église, dont les disciples ou les adhérents respecteraient un credo ; à cette foule de seconds rôles, il oppose l'unicité d'un protagoniste, le regretté Walter Burkert, qui n'aurait pas fondé une « école » et dont l'ampleur des intérêts scientifiques ne se laisserait pas réduire à une étiquette simpliste. Je ne m'attarderai pas à démanteler dans les détails ces affirmations de Henk Versnel, dont chaque lecteur avisé est en mesure de reconnaître la portée caricaturale. Ce qui m'intéresse ici, c'est d'attirer l'attention sur le fait que l'«École de Paris» est une création historiographique qui fonctionne souvent en tant qu'«idole polémique » et aussi que cette image réductrice est l'un des termes d'un paradoxe dont l'ouvrage de Versnel est un exemple éclairant. Bien qu'elle soit précédée par des déclarations de principe qui sont vouées à démarquer sa position par rapport à Vernant et à ceux qu'il conçoit comme ses acolytes, l'analyse de la religion grecque antique que le savant néerlandais y déploie leur doit énormément. Car les progrès considérables qu'a connus ce domaine spécifique de recherche au cours des dernières décennies, et auxquels Versnel a aussi contribué pour sa part, la communauté scientifique internationale n'aurait tout 
simplement pas pu les accomplir si les sciences de l'Antiquité dans leur ensemble n'avaient pas accueilli, à tous les niveaux, l'influence de l'anthropologie historique qui en a renouvelé les questionnements et les méthodes, avec plus ou moins de résistance et/ou de conscience suivant les différents champs disciplinaires. Ce renouvellement bénéfique, dont les membres du Centre Louis Gernet (de recherches comparées sur les sociétés anciennes) ont été, non pas les seuls acteurs, mais parmi les acteurs principaux, s'est également traduit par une ouverture jusque-là inédite des études classiques à d'autres branches du savoir et à un plus large public, ainsi que par un remarquable succès éditorial de leurs travaux, en France comme à l'étranger. L'« infection » a fini graduellement par prendre racine au cœur même de l'antiquisantlambda (et sans doute même de l'antiquisant-alpha), et elle en a changé le code génétique pour toujours, au point que cette mutation n'est plus ressentie comme telle de nos jours. Les mouvements de balancier font partie intégrante de la vie académique, d'autant plus qu'ils peuvent s'avérer utiles aux savants en quête d'affirmation identitaire, et c'est sans doute à l'une de ces oscillations périodiques que se rattache la fabrique de l'«École de Paris». Je ne saurais exclure que la création de cet «idolo polemico " puisse au final se révéler très utile, s'il s'avère qu'elle nous aura poussés à remettre en question nos certitudes, à argumenter nos positions, à affiner une méthode d'étude, à sélectionner ce qu'il faut retenir de l'héritage de nos devanciers et ce qui, dans leurs ouvrages, paraît caduc au vu des nouvelles acquisitions de la recherche, bref à aller de l'avant. Je suis sûre, en revanche, qu'une perspective historiographique lucide en cette matière est indispensable, qu'il faut essayer de reconsidérer le passé récent de nos études sine ira et studio, sans se faire ni les inquisiteurs ni les apologistes d'une prétendue "Église» parisienne (ou vernantienne). Il est temps peut-être aussi de retirer le structuralisme de la liste des crimes commis contre l'humanité, pour le reconsidérer sereinement, à partir d'une perspective historiographique, en mettant ainsi un terme à une aversion irrationnelle et anachronique qu'on rencontre tout particulièrement chez les historiens et les philologues de l'Antiquité en mal de passé.

2 Étant donné mes racines italiennes et mon domaine de recherche, à savoir l'anthropologie religieuse de la Grèce ancienne, j'aurais pu céder à la tentation d'un exercice de comparaison entre l'«École de Paris» et la "Scuola romana», dans la mesure où l'enseignement d'Angelo Brelich en Histoire des Religions à l'Université « La Sapienza » se déroule à peu près dans les mêmes années et sur des thèmes en partie voisins. Je me limiterai ici à signaler que les deux milieux n'ont interagi que de manière ponctuelle, et c'est donc à distance, en parallèle et chacun à sa manière, qu'ils ont contribué au balisage, dans les études sur les religions antiques, d'un nouveau champ d'enquête, centré sur le polythéisme et la méthode adéquate pour l'analyser. L'une des étapes de ce parcours passe par Rome, comme en témoigne la version rédigée du cours tenu par Angelo Brelich entre 1957 et 1958 à l'Université «La Sapienza », mais publiée seulement il y a une dizaine d'années. Ce petit volume (Brelich 2007), qu'il faudrait intégrer à toute réflexion historiographique sur la religion de la Grèce ancienne, est d'autant plus précieux qu'il permet de prendre place dans la salle de cours de Brelich et d'en "écouter » presque les réflexions sur le polythéisme, sujet primordial, mais presque inexploré à l'époque. Ce qui apparaît comme novateur, par rapport aux historiens des religions qui l'ont précédé, c'est surtout le questionnement choisi, qui essaie d'identifier la spécificité de la forme religieuse dénommée "polythéisme », sans la subordonner à la question du monothéisme, et interroge la manière dont les divinités au pluriel se structurent en un panthéon. Même si certains des points de 
départ et des conclusions de Brelich ne sont plus d'actualité, les réflexions qu'il proposait à ses étudiants sur plusieurs aspects des religions antiques gardent aujourd'hui encore un grand intérêt. Surtout, elles ont contribué à la véritable découverte du polythéisme en tant qu'objet d'étude, alors qu'il avait d'abord été considéré comme une forme primitive ou dégénérée de religion, et ensuite comme une juxtaposition de plusieurs divinités, agencées entre elles au gré des circonstances. Les traces d'une telle attitude n'ont pas complètement disparu de nos jours. Néanmoins, un changement considérable s'est produit dès que la communauté des chercheurs a commencé à envisager de concert les dieux en tant que constructions culturelles complexes et à prendre au sérieux la " société » particulière qu'ils constituent.

Un tel changement est solidaire d'un chemin dont certaines étapes essentielles sont associées aux figures de savants tels que Louis Gernet, Georges Dumézil, Jean-Pierre Vernant, Marcel Detienne, mais cette lignée de "pères fondateurs ", si noble soit-elle, ne suffit pas à en rendre raison. Ce qu'on oublie trop souvent, c'est le remarquable travail collectif qui a caractérisé les recherches sur l'Antiquité conduites pendant plusieurs années au Centre Louis Gernet. Les progrès qui ont été accomplis dans ce milieu et en dialogue avec lui, en France comme à l'étranger, quant à l'étude de la religion grecque antique et du polythéisme en particulier, sont indissociables d'une démarche collective, marquée par une perspective anthropologique, qui a investi à la fois l'analyse des pratiques sociales et des représentations figurées, les démarches des historiens, les procédures et le questionnement des archéologues, l'interprétation des textes et des formes poétiques, bref tout un travail d'équipe qui a été ultérieurement stimulé par la mise en place d'ateliers comparatistes. Or, les sociétés de la Méditerranée ancienne connaissent une imbrication particulièrement étroite entre la religion et les multiples aspects de la vie culturelle, sociale, économique et politique, de sorte qu'il n'est pas pertinent de postuler, dans ces contextes, une sphère religieuse séparée de, et opposée à, une sphère qui ne le serait pas, que l'on pourrait qualifier de «profane». C'est pourquoi l'anthropologie religieuse a contribué de manière significative à la compréhension d'ensemble de ces sociétés dans les différentes phases de leur histoire, permettant de saisir et d'apprécier dans leurs interactions mutuelles les divers aspects d'une culture donnée. C'est aussi pourquoi l'étude de la religion grecque antique, et du polythéisme en particulier, a trouvé dans de telles recherches collectives et pluridisciplinaires un terrain propice à son renouvellement.

4 Ce que les historiens des religions oublient le plus souvent en employant l'étiquette «École de Paris ", c'est qu'il ne s'agit pas du tout d'une école d'histoire des religions à proprement parler, comme l'a été en revanche la «Scuola romana ». Cette impression trouve une justification dans les liens étroits qui ont toujours existé entre le Centre Louis Gernet et la section de Sciences religieuses de l'École Pratique des Hautes Études. La situation était fort différente en Italie, et même dans d'autres pays, où une discipline dénommée Histoire des Religions a eu bien plus de place qu'en France dans les universités mêmes. Et si j'en parle au passé, surtout en ce qui concerne l'enseignement de cette discipline dans l'Université italienne, c'est à bon escient. Le fait est que, dans un milieu académique habitué à reconnaître et à valoriser plutôt la verticalité des relations maître(s)-élèves que l'horizontalité de la coopération scientifique, les figures de Jean-Pierre Vernant et de Marcel Detienne, leurs domaines de recherche, sans oublier le succès éditorial de leurs ouvrages et leur rayonnement international, ont sans aucun doute contribué à l'identification entre l'« École de Paris » et une démarche tournée prioritairement vers l'étude des religions antiques, des dieux et des «mythes ». 
De mon point de vue, au contraire, l'application la plus correcte de ce label inadéquat serait celle qui associerait l'« École de Paris » à l'étude de l'Antiquité, tous domaines confondus, dans une perspective d'anthropologie historique. Mais tout est, précisément, question de perspective.

5 Dans la perspective qui était la mienne à l'université de Naples, pendant mes études au début des années 1990, quand il existait une discipline dénommée précisément "Religioni del mondo classico» (disparue depuis en faveur de l'histoire des religions tout court, maintenant en voie de disparition à son tour), moi aussi je regardais Paris comme le berceau du renouveau en matière de religions antiques. Ce n'est qu'une fois que j'ai commencé à étudier à Paris même, et à fréquenter de manière conjointe l'EPHE et le Centre Gernet, que j'ai pu faire l'expérience concrète d'une pratique interdisciplinaire qui a profondément marqué ma formation. Je n'ai jamais été structuraliste, mais je suis devenue, à ma manière certes, anthropologue dans l'étude des religions antiques. Aucune profession de foi ne m'a été demandée, et du reste je ne l'aurais pas admis. Comme bien d'autres collègues qui appartiennent à la même génération que moi et qui fréquentaient le laboratoire de la rue Monsieur-le-Prince, j'ai été influencée par un certain "esprit Gernet » qui s'est traduit, pour moi, avant tout dans une liberté totale et bénéfique quant à la démarche à suivre pour appréhender mon objet. J'ai choisi mon sujet de thèse doctorale, j'ai été la seule responsable de la méthode adoptée pour l'étudier, même si elle eût sans doute été différente dans un autre milieu, dans la mesure où j'ai été particulièrement sensible à une certaine manière d'étudier le polythéisme et d'envisager les cultures antiques. Cela pour dire que l'« École de Paris » je ne sais pas ce que c'est: ce n'est ni ma «religion », ni mon «idolo polemico ». Et mon cas est loin d'être isolé. Je sais en revanche que, pendant un long moment de ma formation, j'ai entretenu un dialogue libre et fécond avec les ouvrages de Vernant et de Detienne, que j'ai essayé, et que j'essaie encore aujourd'hui, de faire la part des choses entre ce que je retiens de leurs enseignements et ce qui me paraît devoir, et pouvoir, être revu. Et mon cas est loin d'être isolé. Ce que l'étiquette «École de Paris » étouffe, c'est, avant tout, la variété et la richesse des biographies intellectuelles de celles et ceux qui ont participé, dès sa fondation, aux recherches collectives menées au Centre Gernet. Ce qu'elle néglige aussi, c'est le chemin parcouru depuis, dans la mesure où le label en question fige dans le temps un milieu scientifique mouvant, qui s'est élargi et a changé ${ }^{2}$. Sans doute l'obstination à écrire dans des langues autres que l'anglais y est-elle pour quelque chose.

Puisqu'il n'y a pas de règles sans exception, Robert Parker, par exemple, paraît mieux disposé que la plupart de ses collègues à prendre en compte cette complexité quand il soumet à une critique, même sévère, ce qu'il définit comme "the structuralist approach» dans l'analyse des dieux de la Grèce ancienne (Parker 2011, p. 84). Sans entrer dans le détail de sa critique et d'une telle analyse, qui ne sont pas l'objet direct de ces quelques réflexions, il est intéressant de remarquer que la définition de l'objet (l'approche structuraliste plutôt que l'« École de Paris ») correspond à un élargissement de la perspective et qu'à côté des ouvrages les plus célèbres de Vernant et de Detienne trouvent place des travaux plus récents, associés par l'historien à cette même approche. D'un côté, il défend l'incohérence du polythéisme hellénique face à ce qu'il perçoit comme une tentative de lui imposer un ordre artificiel de l'extérieur, de l'autre, il affirme que «the best model to think with or against in analyzing the gods is that offered by structuralism» (Parker 2011, p. 88). Le scepticisme surveillé que Parker applique à la critique de ce modèle (et de tout modèle) cohabite ainsi avec l'emploi de 
ce dernier à des fins exploratoires et avec la conscience que l'état actuel de la recherche en ce domaine ne peut pas en faire l'économie. J'espère avoir bientôt l'occasion d'argumenter les divergences qui existent entre l'analyse qu'il fait de ce modèle en particulier et l'évaluation qui est la mienne du passé récent de la recherche dans ce même domaine, mais j'aimerais souligner dès à présent que des acquis communs existent également et que la constitution d'un tel savoir partagé est la conséquence, qu'on le veuille ou non, de cette mutation profonde dont je parlais auparavant, qui a conduit bon nombre de savants à considérer le polythéisme et son fonctionnement en tant qu'objet d'étude et à repenser la manière d'interroger les dieux des Grecs et des Romains.

7 À partir de l'intuition de Louis Gernet qu'un dieu serait un "système de notions ", et des études de Georges Dumézil, avec l'introduction de la notion de mode d'action, un long parcours a conduit à remettre en question tout modèle statique de panthéon et à repenser les divinités et les groupements qu'elles constituent en fonction de leur capacité à se décliner et à se « ré-agencer » suivant les différents contextes. En ce qui me concerne, je ne crois pas que la souplesse qui caractérise le polythéisme implique nécessairement une incohérence logique de fond et je pense que les incohérences ponctuelles qu'on croit déceler sont le plus souvent à mettre sur le compte de notre propre défaut de compréhension et/ou de connaissance. Comme le dit fort à propos Versnel (2011, p. 84) «whoever finds chaos has been on the lookout for structure». Plutôt que de partir à la recherche des structures figées du polythéisme pour en conclure qu'il serait fondamentalement chaotique, mon opinion est qu'il faut l'aborder comme un langage et essayer d'en comprendre le sens. J'ai l'intention d'argumenter cette conviction dans un ouvrage en préparation, provisoirement intitulé Le langage du polythéisme, qui devrait se composer aussi d'une partie historiographique où seront développées les réflexions préliminaires présentées ici.

En attendant, j'ai choisi de verser à ce dossier consacré à l'« École de Paris » un court texte que j'ai présenté il y a une dizaine d'années à une journée d'études en mémoire de Jean-Pierre Vernant et Pierre Vidal-Naquet ${ }^{3}$. Ce n'est pas un essai scientifique à proprement parler, mais un témoignage personnel du dialogue que j'ai entretenu, dans mes années de doctorat, avec l'une des plus célèbres études de Vernant sur les dieux grecs. Je le livre aux lecteurs tel quel, sans y introduire les modifications qui seraient nécessaires si je voulais qu'il adhère à l'état actuel de mes recherches dans ce domaine ${ }^{4}$. Il photographie une étape de mon parcours qui, pour appartenir au passé, ne fait pas moins partie intégrante du chemin accompli depuis et qui en a indiqué la direction. Dans le cadre présent, j'espère qu'il saura illustrer, par un exemple concret, que la relation à un héritage d'un chercheur en formation peut être autre chose que l'adhésion à une quelconque Église.

\section{Invitation au voyage. Autour d'Hestia-Hermès (Paris, 19 octobre 2007)}

9 Pour commencer, une remarque d'ordre général, en tableau de fond. Les figures diverses sous lesquelles les Grecs se représentent leurs dieux, et les associations par lesquelles ils en articulent les compétences, constituent d'importants objets d'étude qui permettent d'envisager la façon grecque de penser le divin au pluriel et d'établir des relations entre les différents ordres de puissances à l'œuvre dans l'univers. Les 
divinités ne sont pas des coquilles vides, ni leurs théonymes des contenants informes, dont seul le jeu des circonstances déterminerait le contenu. Si, et je cite, «les dieux helléniques sont des puissances, non des personnes ${ }^{5}$ " (Vernant 1965a, p. 274), cela ne signifie pas qu'ils soient interchangeables, bien au contraire. Les Grecs n'ont pas bâti d'innombrables temples, agencé des autels et des dispositifs cultuels, parfois très complexes, juste pour faire joli. Tout cela n'est pas gratuit. Même si le langage polythéiste ne nous est pas toujours accessible, il faut au moins être conscient du fait que ses articulations ont du sens. Négliger ce langage et ce sens constitue, pour l'historien comme pour l'anthropologue, une faute de méthode.

10 C'est ce même langage polythéiste qui, à mon sens, est au cœur de l'étude que Jean-Pierre Vernant a consacrée à Hestia et Hermès, une étude qui a largement contribué à renouveler l'approche de la religion grecque (Vernant 1965b); sur un plan personnel, cette lecture a sans aucun doute contribué à déclencher un questionnement sur le fonctionnement du polythéisme et les représentations des dieux, questionnement qui continue d'orienter mon voyage en pays grec.

11 Je me suis retrouvée souvent à dialoguer avec ces pages de Mythe et pensée qui s'ouvrent sur l'analyse d'un couple de divinités. Et c'est de l'état actuel de ce dialogue que j'aimerais vous parler brièvement aujourd'hui. Le point de départ de mes recherches sur Aphrodite a été son association récurrente avec Arès dans les récits et les cultes (Pironti 2005). Bien évidemment, ce n'est qu'à l'issue d'un long apprentissage que j'ai " abouti », pour ainsi dire, à ce point de départ.

12 Il n'y a pas si longtemps la religion grecque était abordée comme s'il s'agissait d'une série de monothéismes juxtaposés. Depuis, nous sommes un certain nombre à avoir appris qu'il faut étudier les polythéismes anciens en respectant leur structure à dieux multiples. C'est un acquis du point de vue théorique, même si, aujourd'hui encore, il n'est pas évident de trouver, parmi les Antiquisants, des praticiens de cette théorie.

Dans son étude sur Hestia et Hermès, publiée une première fois en 1963, Vernant applique, et adapte, à la religion grecque l'analyse des structures panthéoniques préconisée par Dumézil. Il ne part pas à la recherche d'une structure englobante de type trifonctionnel, dont Dumézil lui-même ne trouvait que peu de traces en Grèce. Il se concentre sur une articulation précise, à deux divinités: à partir de l'association d'Hestia avec Hermès sur la base de la statue de Zeus à Olympie, sculptée par Phidias, il réfléchit sur le sens de ce couple divin dans la tradition et la pensée des Grecs.

De même que les divinités ne sont pas des personnes, ni de simples personnages, mais des puissances divines, leurs associations ne sont pas des artifices, mais ont bien un sens. Ce type d'approche, visant à dépasser une perspective strictement monographique pour atteindre autrement les noyaux sémantiques du panthéon, constitue une avancée considérable vers une meilleure compréhension du polythéisme hellénique.

Hestia-Hermès, une déesse et un dieu, mais ni mari et femme, ni frère et sœur, ni mère et fils; un couple, mais pauvre en récits et en arrière-plan mythique. Il se prête alors particulièrement bien à une analyse ancrée dans l'affinité fonctionnelle. Vernant interroge les rapports de ces deux puissances divines à la lumière de l'organisation de l'espace et du mouvement. Et c'est en s'attachant à cette relation fonctionnelle qu'il arrive à mieux cerner leurs spécificités respectives : du côté d'Hestia, le foyer, le centre, la permanence ; du côté d'Hermès, le seuil, les frontières, le mouvement, le changement 
et l'échange. Et je cite : «à Hestia, le dedans, le clos, le fixe, le repli du groupe humain sur lui-même ; à Hermès, le dehors, l'ouverture, la mobilité, le contact avec l'autre que soi » (Vernant 1965b, p. 101). Cette lecture de l'association Hestia-Hermès débouche sur une explication de leurs fonctions en termes de polarité et de complémentarité. La logique de l'opposition complémentaire, d'inspiration structuraliste, trouve ici l'une de ses applications les plus heureuses.

Mais Hestia-Hermès est surtout le réactif qui lui donne accès à la façon grecque de penser l'espace de l'oikos et celui de la cité. Vernant part des dieux pour aller ailleurs ; des représentations divines et spatiales, il passe ainsi aux pratiques sociales et institutionnelles dont ces représentations lui paraissent solidaires. Et ce sont des pages remarquables, justement célèbres. À partir d'Hestia et de l'hestia, le foyer, il réfléchit sur le symbolisme de ce dernier, sur la lignée, le mariage, les rôles respectifs de l'homme et de la femme, l'épiclérat, et puis les Amphidromies, ces rites de naissance qui rattachent le nouveau-né au foyer domestique. Depuis la scène tragique, les figures d'Oreste, d'Électre, d'Égisthe et de Clytemnestre, appelées autour du foyer, y reçoivent un nouvel éclairage.

Vernant le dit en toutes lettres : dans cette étude, il y a beaucoup plus d'Hestia que d'Hermès, et Hermès n'est envisagé que dans la perspective d'Hestia. Le choix d'un fil conducteur, qu'il soit une divinité ou un domaine, finit souvent par s'imposer d'une manière ou d'une autre, car c'est pragmatique, opératoire, dans la recherche, comme dans l'exposé de ses résultats: l'Apollon de Detienne en est un autre exemple (Detienne 1998). Je crois que l'essentiel n'est pas de renoncer à ce fil conducteur ; ce qui est, en revanche, indispensable est bien de dépasser la perspective monographique, de ne plus étudier les divinités de manière isolée, mais de les replacer, dès la phase préliminaire de l'enquête, à l'intérieur d'un réseau, d'une configuration, d'un panthéon ${ }^{6}$.

18 C'est ce que j'ai essayé de faire, dans mes recherches sur Aphrodite : c'est à partir de son association avec Arès, c'est en réfléchissant sur ce couple divin, que j'ai appris à regarder Aphrodite autrement, ce qui m'a permis d'envisager d'autres aspects de la déesse grecque. Aphrodite et Arès, encore une déesse et un dieu, mais qui constituent cette fois un «vrai » couple : dans les récits mythiques, ils sont amants, mariés, alliés ; de plus, leur union produit aussi une descendance. C'est donc un autre cas de figure par rapport à Hestia-Hermès. L'arrière-plan mythique est fort, voire tellement fort qu'on lui a souvent subordonné les nombreuses attestations cultuelles du couple.

Comme dans le cas d'Hestia-Hermès, cette association n'est pas qu'un artifice littéraire, mais une structure, une articulation panthéonique porteuse de sens. Oui, mais lequel? Quand j'ai commencé à me pencher sur cette question, la réponse habituelle se fondait sur la complémentarité des opposés, le modèle Hestia-Hermès ayant fait école: à Aphrodite, l'amour, la paix, la féminité pure, le mariage, la sphère privée, l'intérieur, la faiblesse ; à Arès, la haine, la guerre, la virilité pure, les armes, le monde extérieur, la force physique. Et comment expliquer alors les cultes en l'honneur d'une Aphrodite armée et les honneurs que lui rendent les militaires, même indépendamment d'Arès? Le renvoi à l'Orient pour expliquer ces cultes bien grecs ne me satisfaisait pas. Il m'est apparu évident que la question fondamentale était de comprendre le rapport d'Aphrodite à la fois avec Arès et l'univers de la guerre, et que ces deux dossiers n'en faisaient qu'un. 

Quand j'ai commencé à étudier les dieux des Grecs, j'ai appris que, pour cerner la spécificité d'une puissance divine, il fallait en identifier moins le domaine d'intervention que le mode d'action, et que la complémentarité des opposés était l'une des clés pour comprendre les structures du panthéon. Chemin faisant, j'en suis arrivée à d'autres "principes ", fussent-ils provisoires : la spécificité d'une divinité est moins dans son mode d'action que dans le réseau souple et cohérent que ses modes d'action et ses lieux d'intervention dessinent ; quant à l'opposition complémentaire, elle est certes une possibilité heuristique, mais pas une règle. Ainsi, elle ne suffit pas à expliquer le couple Aphrodite-Arès, dont les domaines se recoupent et se croisent plus souvent qu'ils ne s'opposent.

21 Concernant ce couple, la réponse habituelle, qui est aussi celle de Vernant, est profondément influencée par l'image d'une Aphrodite toute tendresse et douceur, incarnation de l'éternel féminin, déesse de l'amour. Maintenant on peut en sourire, mais il ne faut pas sous-estimer la puissance et la persistance de cette figure dans notre imaginaire. «Aphrodite, écrit Vernant, n'a pas pour apanage la domination violente, la contrainte physique propres aux divinités guerrières" (Vernant 1965b, p. 102). Pourtant, j'ai pu constater que violence, domination, contrainte et corporéité sont bien présentes dans les représentations grecques d'Aphrodite. Aussi la redoutable déesse qui préside à la mixis, la rencontre des corps, et à l'éros, la pulsion vers l'autre, a-t-elle toute sa place aux côtés d'Arès dans les panthéons des cités grecques, et peut-elle être elle-même appelée à intervenir sur le champ de bataille . Vernant écrit : « Arès et Aphrodite, Polémos et Philia, Neikos et Harmonia, Éris et Éros, apparaissent dans les structures du panthéon, les récits légendaires, les théories des philosophes, comme des couples de puissances, opposées mais étroitement unies, présidant à ces institutions complémentaires que forment la guerre et le mariage " (Vernant 1968, p. 15). Cette lecture en termes d'opposés complémentaires, qui était dans l'air du temps, présuppose le portrait canonique d'Aphrodite et le remet au goût du jour. Elle puise aussi à la conviction profonde que la polarité est constitutive de la pensée grecque.

23 En parcourant la documentation ancienne concernant Aphrodite, j'ai pu mesurer la distorsion introduite par l'image, si chère à la tradition occidentale, de la « déesse de l'amour ». Et j'ai pu mesurer aussi les limites des oppositions binaires masculinféminin, extérieur-intérieur, public-privé, guerre-paix et ainsi de suite, face à la polyvalence des dieux grecs. Contrairement aux idées reçues, qui la présentent comme une déesse des femmes, Aphrodite est hautement concernée par les hommes et la puissance virile; loin de rester confinée dans le gynécée, elle peut prendre place au cœur de la cité et déployer en sa faveur des compétences d'ordre politique et militaire.

24 Certes, si je suis arrivée à ces conclusions, c'est que j'ai fait plus de place aux données cultuelles; c'est dans l'air de notre temps. Et j'ai appris qu'« une déesse n'est pas une femme » (Loraux 1991), ce dont j'ai pu constater le bien-fondé. Il est toujours délicat de projeter ce que l'on croit connaître de la société des hommes sur une société, si spécifique, comme celle des dieux. Pour le dire avec les mots de Vernant: «la pensée religieuse obéit à des règles de classification qui lui sont propres» (Vernant 1965b, p. 101). J'ajouterai que, lorsqu'on se tourne vers les représentations des puissances divines, il est essentiel de prendre en compte ce décalage. 

y a Hestia, mais aussi des Zeus domestiques, le Zeus Ktêsios et l'Herkeios. En outre, la parthenia divine ne se conjugue pas systématiquement avec fixité et permanence dans l' oikos; il suffit de penser à Artémis, que l'on aurait du mal à enfermer à l'intérieur. Le dehors et la mobilité, quant à eux, ne sont pas non plus l'apanage des seules puissances masculines. Aphrodite partage avec Poséidon des compétences sur le grand large, et c'est surtout à la déesse que s'adressent les Grecs lorsqu'il est question de voyager à travers l'espace maritime ${ }^{8}$. De même, mouvement, échange et changement dessinent une intersection entre Aphrodite et Hermès. Tout cela pour dire qu'il faudrait sans doute introduire des nuances à la table des oppositions utilisée par Vernant pour étudier Hestia et Hermès.

ins, il est incontestable que, dans le cas d'Hestia, le statut virginal se conjugue avec fixité et permanence dans l'oikos. Mais c'est Hestia qui est représentée de cette façon-là. L'analyse que Vernant a faite de cette divinité reste donc pertinente. Et la logique des opposés complémentaires se révèle, dans le cas précis d'Hestia-Hermès, un outil adapté pour faire ressortir les relations que ces dieux-là entretiennent respectivement avec l'organisation de l'espace et le mouvement. En revanche, l'application systématique et généralisée, aux autres articulations du panthéon, de cette grille de lecture, n'est pas pertinente. Dans le cas d'Aphrodite-Arès, loin d'être une aide, elle est même un obstacle.

En conclusion de son étude, Vernant écrit : «Encore faut-il indiquer que la polarité, marquant sur tous les plans les rapports de la déesse avec Hermès, est un trait si fondamental de cette pensée archaïque qu'on la retrouve à l'intérieur même de la divinité du foyer, comme si nécessairement une part d'Hestia appartenait déjà à Hermès » (Vernant 1965b, p. 140). Il s'en explique quelques pages auparavant : «La complémentarité des deux divinités suppose, en chacune d'elles, une opposition ou une tension intérieure qui confère à leur personnage de dieu un caractère fondamental d'ambiguïté » (Vernant 1965b, p. 117).

Je me trompe peut-être, mais je crois déceler là un doute, une nuance, un glissement. $\mathrm{Ce}$ caractère fondamental d'ambiguïté, qui est un élément essentiel de la représentation grecque du divin, Vernant le perçoit clairement, mais n'en rend pas compte; dans le modèle descriptif qu'il propose - la part d'Hermès à l'intérieur d'Hestia - c'est une fois de plus la polarité qui prévaut. Sans doute, pour rendre aux dieux toute leur " ambiguïté », d'autres types de relations que la polarité restaient-ils à explorer.

Bref, une fois le tableau de fond posé, le voyage continue.

\section{BIBLIOGRAPHIE}

Bonnet C. (2017), compte rendu de : Eidinow, Kindt et Osborne 2016, BMCR 2017.06.13. 
Bonnet C. et al. éd. (2017), Puissances divines à l'épreuve du comparatisme. Constructions, variations et réseaux relationnels, Turnhout.

Brelich A. (2007), Il politeismo, éd. M. Massenzio et A. Alessandri, préface M. Augé, Rome.

Detienne M. (1998), Apollon le couteau à la main. Une approche expérimentale du polythéisme grec, Paris.

Eidinow E., Kindt J. et Osborne R. éd. (2016), Theologies of Ancient Greek Religion, Cambridge.

Loraux N. (1991), « Qu'est-ce qu'une déesse ? », dans Duby G. et Perrot M. dir., Histoire des femmes. L'Antiquité, vol. I, éd. P. Schmitt Pantel, Paris, p. 31-62.

Parker R. (2011), On Greek Religion, Ithaca (N.Y.)-Londres.

Pirenne-Delforge V. et Pironti G. (2016), L’Héra de Zeus. Ennemie intime, épouse définitive, Paris.

Pironti G. (2005), « Aphrodite dans le domaine d'Arès. Éléments pour un dialogue entre mythe et culte ", Kernos 18, p. 167-184.

- (2007), Entre ciel et guerre. Figures d'Aphrodite en Grèce ancienne, Liège.

- (2015), « Da Poseidone ad Afrodite : riflessioni preliminari sul pantheon marittimo nella Grecia antica », SMSR 81-1, p. 165-178.

Vernant J.-P. (1965a), «Aspects de la personne dans la religion grecque » dans Id., Mythe et pensée chez les Grecs, Paris, p. 267-282.

- (1965b), «Hestia - Hermès : sur l'expression religieuse de l'espace et du mouvement chez les Grecs ", dans Id., Mythe et pensée chez les Grecs, Paris, p. 97-143.

- (1968), «Introduction », dans Id. dir., Problèmes de la guerre en Grèce ancienne, Paris, p. 11-38.

Versnel H. S. (2011), Coping with the Gods. Wayward Readings in Greek Theology, Leiden-Boston.

\section{NOTES}

1. Versnel 2011, p. 35 : «A comparison between the very Parisian Religion in the Ancient Greek City by Bruit-Zaidman and Schmitt-Pantel with Burkert's own Greek Religion is indeed illuminating. As for the treatment of the gods, for instance, Burkert adopts the traditional custom of systematically devoting separate treatments to each of the Olympian gods, one after the other. Those Gallic and Gallicizing authors, on the other hand, who follow in the track of the Paris school and hence feel committed to its creed that this is blasphemy, necessarily opt for a different organisation of their books. This principle is now widely accepted, also by scholars of a different denomination. I compared here a book of disciples, or at least adherents, with one of a protagonist. The reason is that, different from the 'École de Paris', one cannot speak of a 'school' of Burkert, inter alia because his scholarly activities display an impressive diversity and his theoretical points of departure cannot be captured under one catchword. »

2. Voir, à ce propos, les justes remarques de Corinne Bonnet (2017). La critique d'une «Paris (or French) school ", image à la fois réductrice et caricaturale, ne peut justifier l'ignorance de la riche bibliographie en langue française des dernières décennies.

3. Autour des œuvres de Jean-Pierre Vernant et de Pierre Vidal-Naquet, Centre Louis Gernet et Équipe Phéacie, 19-20 octobre 2007, INHA, Paris. 
4. La seule actualisation concerne les quelques renvois bibliographiques qui ont été ajoutés à cette occasion.

5. La notion de "puissance divine» est au cœur d'un ouvrage collectif qui en a interrogé les implications diverses dans une perspective comparatiste: Bonnet et al. 2017.

6. L'ouvrage Entre ciel et guerre (Pironti 2007), représentait déjà, dans mes intentions, une tentative en ce sens, que j'ai poursuivie en écrivant, avec V. Pirenne-Delforge, L'Héra de Zeus (Pirenne-Delforge et Pironti 2016).

7. Une affirmation péremptoire telle que « Ad un antico greco non sarebbe mai venuto in mente di chiedere guarigione o prole ad Ares, né una vittoria in guerra ad Afrodite » (Brelich 2007, p. 56) s'est ainsi révélée inexacte. Le démenti offert par le cas d'Aphrodite est intéressant car il montre bien l'obstacle épistémologique que constituent toujours les a priori dans l'appréhension des divinités antiques, dans les années cinquante du siècle dernier comme aujourd'hui.

8. Je me permets de renvoyer à Pironti 2015.

\section{RÉSUMÉS}

Cette contribution se compose de deux volets bien distincts. Le premier est consacré à une révision critique de l'image réductrice de la mal nommée Paris School, en usage encore de nos jours pour définir (et parfois caricaturer) un certain milieu scientifique, au sein duquel l'anthropologie historique des mondes antiques a contribué à faire émerger le polythéisme en tant qu'objet d'étude à part entière. Le second volet consiste en un témoignage personnel, daté de 2007, qui illustre le dialogue de l'auteure avec ce même milieu scientifique, au sein duquel elle s'est formée, et avec une étude particulièrement représentative de Jean-Pierre Vernant sur les dieux grecs.

This contribution consists of two distinct parts. The first is devoted to a critical revision of the reductive image of the misnamed Paris School, still used today to define (and sometimes caricature) a certain scientific milieu, in which the historical anthropology of the ancient world has contributed to the emergence of polytheism as an object of study in its own right. The second part of the contribution consists of a public talk, dated to 2007, which illustrates the author's dialogue with that same scientific milieu, in which she was trained, and with a particularly representative study of Jean-Pierre Vernant on the Greek gods.

\section{INDEX}

Mots-clés : religions antiques, polythéisme, anthropologie, Jean-Pierre Vernant, historiographie Keywords : ancient Religions, polytheism, anthropology, Jean-Pierre Vernant, historiography 
AUTEUR

GABRIELLA PIRONTI

EPHE-PSL, ANHIMA UMR 8210 